\title{
Inpainting Versus Denoising for Dose Reduction in STEM
}

\author{
Toby Sanders ${ }^{1}$ and Christian Dwyer ${ }^{2}$ \\ 1. School of Mathematical and Statistical Sciences, Arizona State University, Tempe, Arizona, USA. \\ 2. Department of Physics, Arizona State University, Tempe, Arizona, USA.
}

In studying beam-sensitive materials using the transmission electron microscope (TEM), minimizing the required dose is usually crucial. There is a recent wave of ideas stemming from the field of image processing, and especially compressed sensing, where, under certain assumptions, one can obtain the same amount of information about the specimen using fewer measurements [1]. For electron tomography, which typically requires a large radiation dose, these concepts have already led to significant improvements in the resolution of the 3D reconstructions [2].

Most attempts at implementing compressed-sensing-related ideas in electron microscopy have been carried out in the STEM geometry. A prevailing idea is subsampling: the image is acquired with the majority of pixels "missing", and then inpainting (interpolation) with a compressed-sensing algorithm is used to recover the image values at the missing pixels [3,4]. Such algorithms can be thought to recover the most likely image given the known pixels by assuming some image regularity in a mathematical sense. If the beam skips $m$ pixels on average, then there is an obvious reduction by $m$ in the total radiation dose compared to acquiring the full number of pixels. The subsampling/inpainting approach is already driving the development of instrumentation [5].

It is also legitimate (not to mention pertinent) to consider reducing the dwell time by the same factor $m$, acquiring the full number of pixels, and then using the same compressed-sensing algorithm. The algorithm becomes a denoising algorithm in this context and its application is consistent with the assumption of a "regular" image. There will be the same obvious reduction in dose, and denoising naturally compensates for the increased noise that usually accompanies a reduced dwell time.

In the present work, we regard the inpainting and denoising approaches as alternative mathematicallybased strategies for reducing the radiation dose. We compare the performance of the two approaches and discuss the circumstances under which one or the other may prevail.

Here we consider the relatively simple case where the only noise is the Poisson noise (other noise contributions are considered later). We use three different test images: the Shepp-Logan phantom, a simulated 2D projection of a piecewise-constant 3D volume, and a simulated crystal diffraction pattern (Figure 1). The original images were completely noise free. We consider images acquired with a fixed total electron dose but different sampling rates (defined as the ratio of sampled to total pixels). Hence for a fixed total dose, greater sampling implies more and noisier pixels in the experiment. The inpainting/denoising techniques implemented followed that of a reweighted L2-L1 minimization to account for Poisson noise statistics [6].

We calculate the relative error between the inpainted/denoised images and the original image (Figure 2). These plots clearly indicate that if the only noise is Poisson noise, then the inpainting approach provides no benefit compared to the "more natural" denoising algorithm. However, the introduction of nonPoisson noise (e.g., "readout" noise) alters these results, as will be discussed. 
References:

[1] E. Candes, and J. Romberg, Inverse problems 23.3 (2007), p. 969.

[2] T. Sanders, I. Arslan, Microscopy and Microanalysis 23.6 (2017), p. 1121.

[3] T. Sanders, C. Dwyer, Ultramicroscopy 182 (2017), p. 292.

[4] A. Stevens et al, Microscopy 63.1 (2013), p. 41.

[5] A. Béché et al, Applied Physics Letters 108.9 (2016), p. 093103.

[6] Li, Jia et al, Inverse Probl. Imaging (Springfield) 9.3 (2015), p. 875.
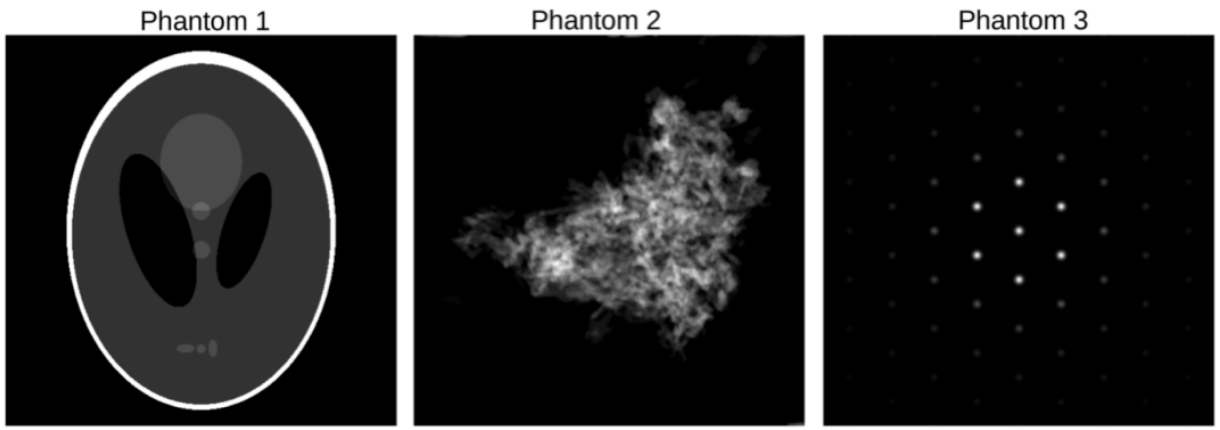

Figure 1. Test images used in the present work.
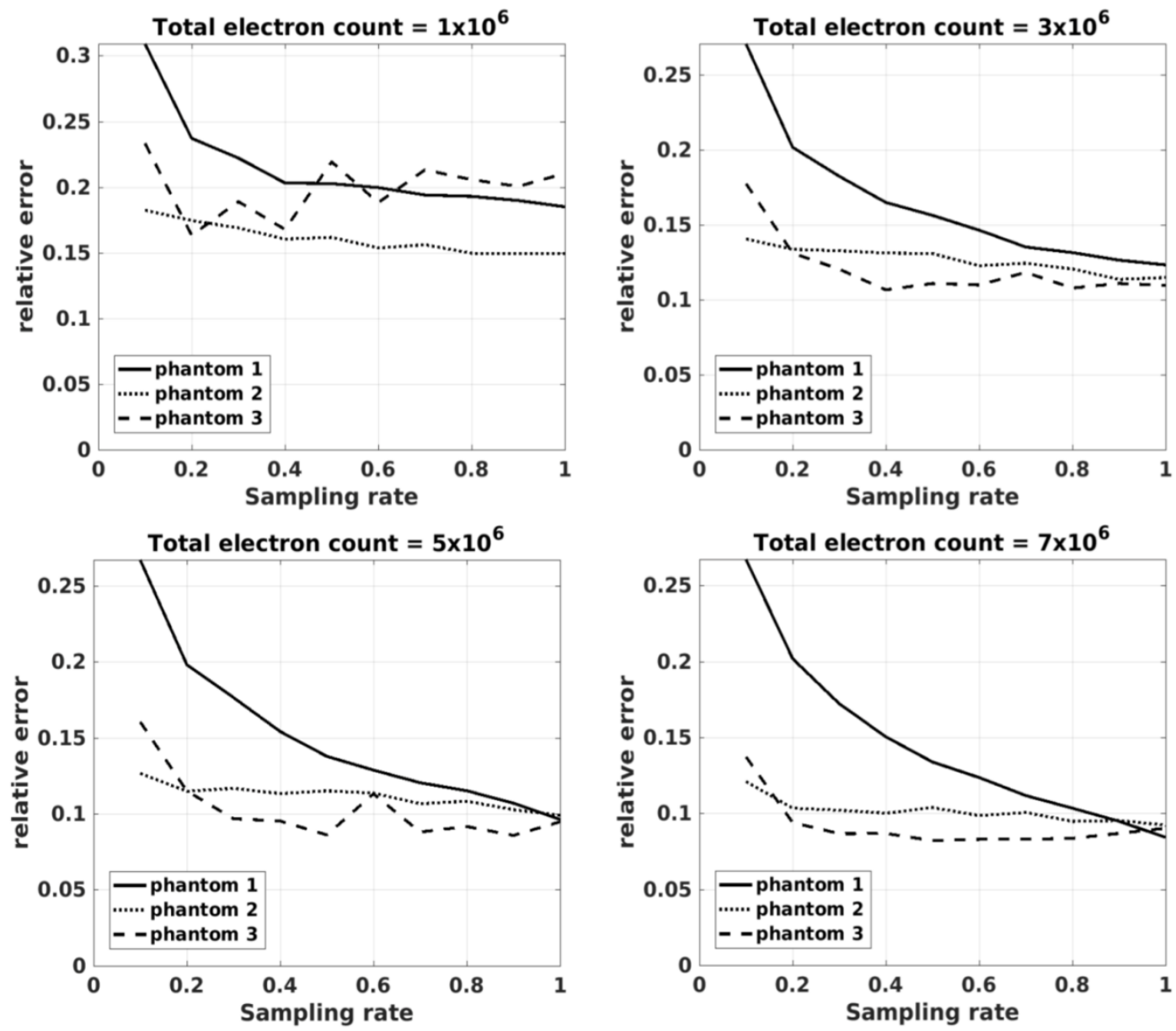

Figure 2. Relative errors between inpainted/denoised images and original images for fixed total electron counts. Sampling rates $<1$ imply inpainting; sampling rate $=1$ implies pure denoising. 